\title{
Isolated interventricular septal tuberculoma causing complete heart block
}

\author{
C WREN, PGI STOVIN
}

From the Regional Cardiac Unit, Papworth Hospital

\section{Case report}

A 66-year-old man, seen in 1971 after a presyncopal episode, was found to have a heart rate of 32 beats per minute. His ECG showed complete heart block with narrow QRS complexes. After two Adams-Stokes attacks in hospital a temporary pacemaker was inserted. He had previously been well with no history of tuberculosis nor of contact with tuberculosis. Investigation

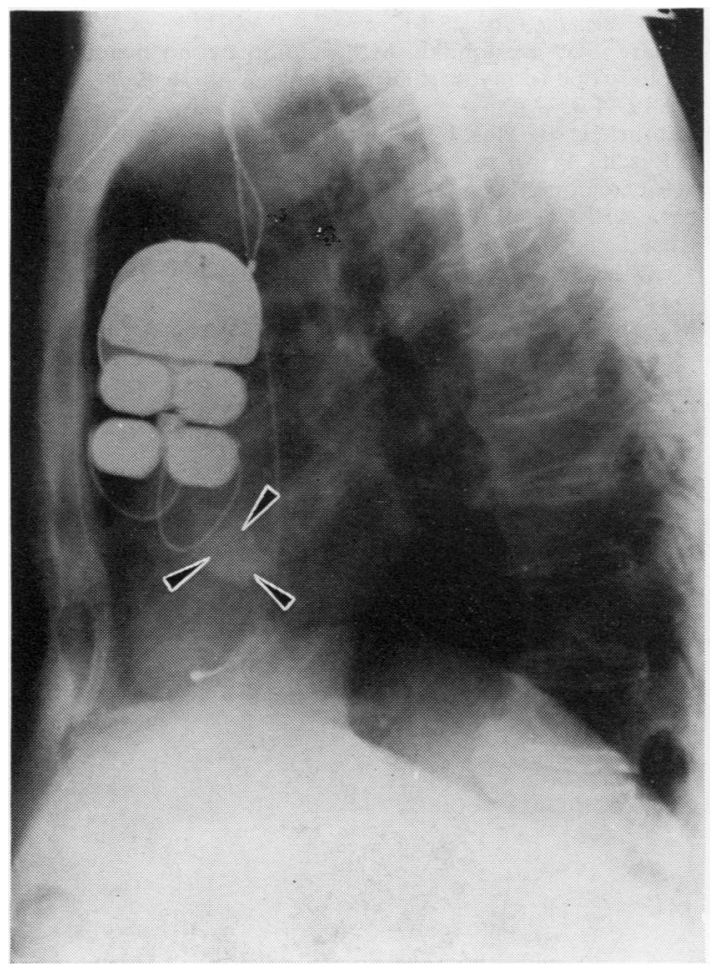

Fig 1 Lateral chest $X$-ray showing area of calcification ( $\rightarrow$ ) within the heart shadow.

Address for reprint requests: Dr C Wren, Regional Cardiac Unit, Papworth Hospital, Papworth Everard, Cambridge CB3 8RE.

showed no evidence of myocardial infarction and the Wassermann reaction was negative.

A permanent pacing system (Devices 3821 generator with an endocardial electrode) was implanted and a postoperative chest radiograph showed a good electrode pesition with no abnormality. The patient remained well and had elective generator changes in 1974 and 1977. On both occasions the lateral chest film showed unexplained calcification within the heart shadow (fig 1).

In 1980 he was admitted for a further elective generator change and was noted to be pale with purpura and splenomegaly. Recent investigation in his local hospital had established a diagnosis of non-Hodgkins' lymphoma with bone-marrow involvement and pancytopenia. A few days after his unit change he developed a fever for which no cause could be found. Despite appropriate treatment with antibiotics his condition deteriorated and he died.

At necropsy the immediate cause of death was found to be a fungal bronchitis. The lymphoma was widespread and involved the bone marrow, intra-abdominal lymph nodes, liver, and spleen. The histological appearances were those of a non-histiocytic non-Hodgkin's lymphoma. There was extensive fibrosis and calcification in the

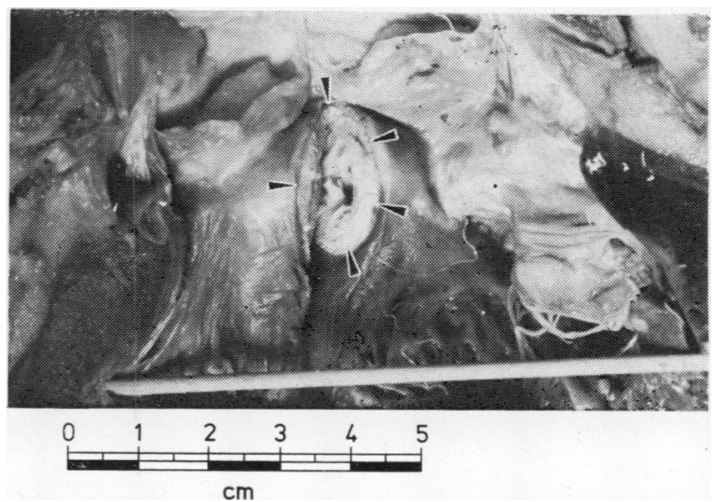

Fig 2 Interventricular septum incised to show the caseous nodule $(\rightarrow)$ with a central hole where a sample had been taken for culture. The tuberculoma extends from the posterior mitral commissure to the centre of the base of the right coronary aortic valve cusp. The aortic valve cusps have been padded out with cotton wool. 
border of a $2 \mathrm{~cm}$ caseous nodule in the crest of the interventricular septum (fig 2) with slight extension into the membranous septum, the ring of the tricuspid valve in the region of the right bundle branch and into the anterior leaflet of the mitral valve. There was no calcification in the mitral valve ring and only a small separate bar of calcium in the left coronary cusp of the aortic valve extending into the anterior leaflet of the mitral valve. The coronary arteries were normal. There was no evidence of active or healed tuberculosis in the lungs or elsewhere.

On microscopy the fibro-caseous nodule showed no cellular reaction but was typical of a calcified tuberculoma. No acid-alcohol fast bacilli were seen and culture was negative. The lesion had completely destroyed the bundle of His but the left bundle branch survived.

\section{Discussion}

Myocardial tuberculosis was rare even in the days before effective anti-tuberculous treatment was available and was usually an incidental finding at postmortem. ${ }^{1}$ In most cases the infection is either part of miliary tuberculosis or an extension of pericardial involvement and in the absence of either of these it is presumed to be secondaary to haematogenous or lymphatic spread. ${ }^{1}$ There is almost always a tuberculous focus elsewhere in the body and isolated tuberculomas are very rare indeed. Atrioventricular (AV) block as a complication of myocardial tuberculosis has been described only rarely ${ }^{2-5}$ and in no case was the cause an isolated interventricular septal tuberculoma. Two of the patients ${ }^{2}{ }^{3}$ had complete heart block with narrow QRS complexes, a third ${ }^{4}$ had 2:1 AV block, and the ECG of the fourth ${ }^{5}$ was not described. Three of the patients ${ }^{245}$ had active tuberculosis elsewhere in the body and in the fourth report ${ }^{3}$ no mention is made of the other postmortem findings. In all cases. as in ours, the final diagnosis was made only at necropsy.

The calcification seen on the lateral chest radiograph in our patient (fig 1) was, in retrospect, caused by the calcified tuberculoma. The size, shape, and position correspond well with the postmortem findings. Calcification visible on chest films has not previously been described in an interventricular septal tuberculoma. There is one report ${ }^{6}$ of a tuberculoma in the left ventricular free wall seen radiographically and although the lesion was not in the septum, asymptomatic first degree heart block was present. In our case the opacity was barely visible in 1974 but increased over the years, becoming obvious by 1980.

At necropsy the only calcification in the aortic valve ran into the anterior leaflet of the mitral valve but did not extend to form a calcific degeneration of the fibrous skeleton of the heart. Such calcific degeneration can form focal expansions of soft calcified material extending into adjacent myocardium from the calcified valve or value ring. ${ }^{\text {? }}$

We thank Dr D Wainwright Evans for permission to report this case and his helpful criticism of the manuscript.

\section{References}

${ }^{1}$ Pomerance A. Tuberculoma of the interventricular septum. Br Heart J 1963;25:412-4.

${ }^{2}$ Kinare SG, Deshmukh MM. Complete atrioventricular block due to myocardial tuberculosis. Arch Pathol 1969; 88:684-7.

${ }^{3}$ Latour H, Baissus C, Nguyen Tan Dong, Grolleau R, Puech P. Bloc auriculo-ventriculaire complet par tuberculome du septum inter-auriculaire. Arch $\mathrm{Mal}$ Coeur 1975;68:315-9.

${ }^{4}$ Rose I. Tuberculosis of the myocardium: report of a case exhibiting changing types of heart block. Am Rev Tuberc $1952 ; 65: 332-8$.

${ }^{5}$ Menon BT, Roa CKP. Tuberculosis of the myocardium causing complete heart block. Am J Pathol 1945;21:11937.

${ }^{6}$ Claiborne TS. Caseating granulomas of the heart. $\mathrm{Am} \mathrm{J}$ Card 1974;33:920-3.

' Pomerance A. Ageing and degenerative changes. In: Pomerance A, Davies MJ, eds. The Pathology of the heart Oxford: Blackwell Scientific Publications, 1975:61-7. 